\title{
Comportamientos intrínseco y extrínseco de piezocerámicas
}

\author{
A. AlbaredA, R. PÉREZ, J.E. GARCíA, D.A. OCHOA
}

Dept. Física Aplicada. Univ. Politècnica Catalunya. E-08034 Barcelona. Spain

\begin{abstract}
Los altos valores de los coeficientes dieléctricos y piezoeléctricos en las piezocerámicas son debidos a la superposición de los efectos intrínsecos y extrínsecos. El objetivo de este trabajo es profundizar en los mecanismos presentes en las cerámicas y que producen estos efectos. Se asume que el efecto intrínseco tiene un comportamiento lineal que es independiente de la temperatura, mientras que el efecto extrínseco, producido por el movimiento de paredes de dominio, tiene una dependencia no lineal y depende de la temperatura. También puede aceptarse que a muy baja temperatura la contribución extrínseca desaparece, debido a que los dominios se congelan. Además se considera que los efectos intrínsecos y extrínsecos son independientes y pueden sumarse. Estas hipótesis permiten calcular los coeficientes lineal, intrínseco y extrínseco. Se han realizado medidas lineales de los coeficientes elásticos, dieléctricos y piezoeléctricos a muy baja temperatura, así como medidas no lineales, sobre diferentes cerámicas. Se analiza la diferente repercusión de las paredes de dominio de $180^{\circ}$ y de no- $180^{\circ}$ en los coeficientes dieléctricos y piezoeléctricos. Se ha desarrollado un modelo de contribución extrínseca por dominios de no- $180^{\circ}$ con estructura laminar de dominios ferroeléctricos en los granos de la cerámica. La anisotropía dieléctrica no lineal, $\left(\Delta \varepsilon_{11}\right)_{\mathrm{NL}}>\left(\Delta \varepsilon_{33}\right)_{\mathrm{NL}}$, se ha medido y confirma dicho modelo.
\end{abstract}

Palabras clave: piezoelectricidad, extrínseco, no linealidad, cerámicas.

\section{Intrinsic and extrinsic behaviours in piezoceramics}

High values of dielectric and piezoelectric coefficients in piezoceramics are due to the superposition of extrinsic and intrinsic effects. The aim of this paper is to gain insights into the intrinsic and extrinsic mechanisms present in ceramics. It is assumed that the intrinsic effect has a linear behaviour and is temperature-independent. On the other hand, the extrinsic effect, which corresponds to the domain wall movement, has a non-linear dependence, and is temperature-dependent. Therefore, it can be assumed that at a very low temperature the extrinsic contribution disappears because the domains freeze up. Also, it is considered that the intrinsic and extrinsic effects are independent and may be added together. From these hypotheses, the linear, intrinsic and extrinsic coefficients may be obtained. Linear dielectric and piezoelectric measurements have been carried out at very low temperatures, as well as non-linear measurements, using different piezoceramic materials. An analysis of the $180^{\circ}$ and non- $180^{\circ}$ domain wall extrinsic contribution is conducted producing different contributions on the dielectric and piezoelectric coefficients. A model of the extrinsic contribution of non- $180^{\circ}$ domain wall in grain ceramics with a ferroelectric domain laminar structure has been developed. The non-linear dielectric anisotropy, $\left(\Delta \varepsilon_{11}\right)_{\mathrm{NL}}>\left(\Delta \varepsilon_{33}\right)_{\mathrm{NL}}$ is measured and supports the previous model.

Key words: piezoelectricity, extrinsic, non-linear, ceramic.

\section{INTRODUCCIÓN}

El estudio de las cerámicas piezoeléctricas de la familia PZT, se ha completado en los últimos años con medidas no lineales. Estas medidas con alta señal han sido necesarias al observar que los comportamientos reales de estas cerámicas se alejaban del comportamiento lineal predicho por las ecuaciones características que rigen a estas cerámicas al trabajar con baja señal. Además este comportamiento no lineal es el que regirá a los dispositivos de potencia, fijando los límites de utilización de muchos de estos dispositivos.

La causa de este comportamiento no lineal hay que buscarla en la naturaleza microscópica de estas cerámicas. La morfología de cada grano de cerámica muestra una configuración de dominios, de forma que en cada dominio todos los dipolos están con la misma orientación. Estos dominios aparecen debido a que estas cerámicas son ferroeléctricas y las orientaciones posibles de los dipolos dentro de cada dominio dependen del grupo cristalino de la cerámica. También, las paredes de dominios, que separan dos dominios colindantes, tendrán restricciones que son función de las condiciones de contorno mecánicas y eléctricas en la frontera de un dominio.

Esta estructura en dominios hace que aparezcan dos tipos diferentes de procesos de cambio en la polarización de un grano cerámico: el efecto intrínseco y el extrínseco. El efecto intrínseco es debido al cambio en la polarización de cada dipolo individual, al cambiar simplemente la magnitud de su polarización, pero sin variar los límites de los dominios ferroeléctricos. Sería el que se produciría si todo el grano 
fuese un único monodominio, cambiando su polarización por efecto del campo eléctrico o esfuerzo aplicado externamente. En contraposición, el efecto extrínseco es el que se produce cuando hay un movimiento en las paredes de dominio, de forma que hay una conmutación de los dipolos al pasar de pertenecer a un dominio a pertenecer al otro dominio vecino. El comportamiento de una cerámica piezoeléctrica es la superposición de ambos efectos, el intrínseco y el extrínseco $(1,2)$. Al aplicar un campo eléctrico menor que el campo coercitivo, $\mathrm{E}<\mathrm{E}_{c^{\prime}}$ como es el caso aquí desarrollado, ciertos fenómenos, como la nucleación y aniquilación de dominios ferroeléctricos, se producen con mucha menos abundancia que cuando se produce la conmutación de la polarización $\mathrm{P}_{\mathrm{s}}$ de toda la cerámica, (que supondría la aplicación de un campo eléctrico mayor que el campo coercitivo $\mathrm{E}_{\mathrm{c}^{\prime}}$ ). Las paredes de dominio vuelven prácticamente a su posición inicial después de un ciclo completo y la estructura de las paredes de dominio apenas varía al realizar un ciclo del campo eléctrico.

En la mayoría de estas cerámicas piezoeléctricas sus elevados coeficientes dieléctricos y electromecánicos provienen precisamente de la contribución extrínseca. Es de destacar que una gran contribución del efecto extrínseco supone un elevado valor para los coeficientes dieléctricos y piezoeléctricos, pero en contrapartida también genera una mayor no linealidad en su comportamiento con alta señal. Es preciso optar por un compromiso en función de la aplicación deseada. Para aplicaciones de potencia se utilizarán cerámicas con menor efecto extrínseco, mientras que para detectores de señal o sensores generalmente interesará materiales con elevados coeficientes y efecto extrínseco. El estudio de estos efectos intrínsecos y extrínsecos es fundamental para entender, diseñar y optimizar el buen comportamiento de una cerámica, no sólo para obtener un elevado valor de los coeficientes dieléctricos y piezoeléctricos, sino también para optimizar las pérdidas dieléctricas o elásticas del material.

A lo largo de este trabajo se va a correlacionar el comportamiento no lineal con el efecto extrínseco, analizando los diversos modelos existentes para las diferentes cerámicas piezoeléctricas. En una segunda etapa, se va a llevar a cabo un estudio de estos comportamientos dieléctricos y piezoeléctricos a muy baja temperatura, próxima a los $0 \mathrm{~K}$ (3). $\mathrm{Al}$ variar la temperatura estos efectos intrínsecos y extrínsecos se comportan de manera muy diferente, de manera que pueden estudiarse separadamente ambos efectos. Esto será importante, pues permitirá analizar y medir cuantitativamente cada uno de estos dos efectos por separado. Por último, se ha desarrollado un modelo, que contempla la morfología observada en múltiples cerámicas, consistente en dominios adyacentes con polarizaciones que cambian $90^{\circ} \mathrm{su}$ vector polarización (o en general un ángulo diferente de $180^{\circ}$ ) separados por paredes de dominio que son planos paralelos. Se estudia como repercute dicho modelo en la anisotropía del efecto extrínseco y se compara con algunas medidas.

\section{COMPORTAMIENTO NO LINEAL}

Analizaremos en este apartado el comportamiento no lineal de las piezocerámicas, el cual depende fundamentalmente del efecto extrínseco del movimiento de las paredes de dominios.

Los comportamientos no lineales que se muestran aquí, pueden ser catalogados en dos categorías y por lo tanto en dos rangos de frecuencias. Por un lado las medidas dieléctricas y piezoeléctricas se llevan a cabo en general a baja frecuencia, típicamente a $1 \mathrm{kHz}$, para evitar el solapamiento con las resonancias. En cambio las medidas de no linealidad elástica, se realizan en la resonancia, que es cuando se producen altos esfuerzos mecánicos y por lo tanto efectos elásticos no lineales.

Las medidas dieléctricas no lineales parten básicamente del estudio de la variación de la permitividad dieléctrica $\varepsilon$ y de las pérdidas dieléctricas tan $\delta_{\mathrm{e}}$ en función del campo eléctrico aplicado:

$$
\begin{aligned}
& \varepsilon-\varepsilon_{L}=f(E) \\
& \tan \delta_{e}=g(E)
\end{aligned}
$$

siendo $\varepsilon_{\mathrm{L}}$ el valor de la constante dieléctrica lineal $\varepsilon_{33^{\prime}}$ o con campo eléctrico aplicado $\mathrm{E}$ pequeño.

El primer modelo utilizado, que proviene del magnetismo, es el modelo de Rayleigh $(1,4)$. Según este modelo, la permitividad dieléctrica aumenta con la amplitud del campo eléctrico aplicado $\mathrm{E}_{0^{\prime}}$ al igual que las pérdidas dieléctricas:

$$
D(E)=\varepsilon_{0}\left[\left(\varepsilon_{L}+\alpha E_{0}\right) E \pm \frac{\alpha}{2}\left(E_{0}^{2}-E^{2}\right)\right]
$$

siendo D el vector desplazamiento eléctrico y E el valor instantáneo del campo eléctrico (5).

Cuando el campo eléctrico aplicado es una función sinusoidal, el desplazamiento eléctrico $\mathrm{D}$ contiene los diferentes armónicos de la frecuencia de la excitación. Al medir la componenteenfasey cuadratura dela componentefundamental de la respuesta, se pueden medir las contribuciones, a esa frecuencia, de la constante dieléctrica compleja $\varepsilon=\varepsilon^{\prime}-\mathrm{j} \varepsilon^{\prime \prime}$. Según este modelo la variación de la constante dieléctrica $\varepsilon^{\prime}$ depende exclusivamente de la amplitud del campo eléctrico $\mathrm{E}_{0}$. Las pérdidas dieléctricas $\varepsilon^{\prime \prime}$ son también proporcionales a $\mathrm{E}_{0} \mathrm{y}$ por lo tanto también al incremento de la constante dieléctrica $\Delta \varepsilon^{\prime}$ :

$$
\begin{aligned}
& \varepsilon^{\prime}=\varepsilon_{\mathrm{L}}+\alpha \mathrm{E}_{0} \\
& \varepsilon^{\prime \prime}=\frac{4}{3 \pi} \alpha \mathrm{E}_{0} \\
& \mathrm{~m}_{\varepsilon}=\frac{\varepsilon^{\prime \prime}}{\Delta \varepsilon^{\prime}}=\frac{4}{3 \pi} \approx 0.42
\end{aligned}
$$

donde el factor $4 / 3 \pi$ proviene de la aplicación de la Ec. [2] para un campo eléctrico sinusoidal, $\mathrm{y}_{\varepsilon}$ es la relación que aparece entre las variaciones de las contribuciones imaginaria y real de la constante dieléctrica.

Este modelo se cumple aceptablemente bien en algunos materiales, correspondiendo a las llamadas cerámicas PZT "blandas", que se utilizan, por ejemplo, como buenos emisores receptores de ultrasonidos.

Sin embargo las cerámicas duras, que poseen pérdidas mucho más pequeñas, no cumplen esta ley de Rayleigh. Para ellas se puede establecer que la variación de la constante dieléctrica $\Delta \varepsilon^{\prime}\left(\mathrm{E}_{0^{\prime}} \mathrm{E}\right)$ depende de la amplitud del campo eléctrico $E_{0} y$ también del valor instantáneo de ese campo 
eléctrico E. Además la dependencia con la amplitud del campo eléctrico $E_{0}$ no es lineal, sino que es, generalmente, una función cuadrática. Se sigue cumpliendo que hay una proporcionalidad entre las pérdidas $\varepsilon^{\prime \prime}$ y la variación de la constante dieléctrica $\Delta \varepsilon^{\prime}$, pero la relación es diferente de la obtenida según el modelo de Rayleigh, obteniéndose valores del orden de $m_{\varepsilon}=0.15$.

En un estudio más detallado de estas contribuciones, se ha descompuesto el incremento de la constante dieléctrica en dos contribuciones $\Delta \varepsilon^{\prime}=\varepsilon_{\alpha}+\varepsilon_{\beta^{\prime}}$ una $\varepsilon_{\alpha}$ que es igual al incremento de la constante dieléctrica a campo eléctrico instantáneo nulo, que depende únicamente de la amplitud del campo eléctrico $\mathrm{E}_{0^{\prime}} \mathrm{y}$ otra contribución $\varepsilon_{\beta}$ que recoge la dependencia instantánea con el campo eléctrico $\mathrm{E}$ :

$$
\begin{aligned}
& \varepsilon_{\alpha}=\Delta \varepsilon^{\prime}\left(0, \mathrm{E}_{0}\right), \\
& \varepsilon_{\beta}(\mathrm{E})=\Delta \varepsilon^{\prime}\left(\mathrm{E}, \mathrm{E}_{0}\right)-\Delta \varepsilon^{\prime}\left(0, \mathrm{E}_{0}\right)
\end{aligned}
$$

Se ha obtenido que la relación entre $\varepsilon^{\prime \prime}$ y $\varepsilon_{\alpha}$ es una relación lineal, $\mathrm{m}_{\alpha}=\varepsilon^{\prime \prime} / \varepsilon_{\alpha}=0.42$, tal como predice el modelo de Rayleigh. Sólo en el caso en que $\varepsilon_{\beta}$ fuese nulo, como prácticamente ocurre en los materiales blandos, se cumpliría que $\mathrm{m}_{\alpha}=\mathrm{m}_{\varepsilon}$. Ello hace suponer que en los materiales duros se están superponiendo dos efectos: un efecto que cumple la ley de Rayleigh y que sería la responsable de las pérdidas (asociado a $\varepsilon_{\alpha}$ ), y otro efecto que crearía la dependencia con el valor instantáneo del campo eléctrico $\varepsilon_{\beta}$, que no está contemplado en el modelo citado. La contribución $\varepsilon_{\alpha}$ se relaciona con procesos irreversibles, mientras que $\varepsilon_{\beta}$ está relacionada con los reversibles.

Un aspecto importante de este análisis es la constatación de que los procesos de envejecimiento de los materiales están íntimamente ligados a esta contribución $\varepsilon_{\alpha^{\prime}}$ habiendo observado como la magnitud $\varepsilon_{\beta}$ no se ve influenciada por dicho envejecimiento (5).

Las medidas del coeficiente piezoeléctrico inverso pueden realizarsesimultáneamentealas medidasnolineales dieléctricas citadas anteriormente. Basta con disponer de un sensor de desplazamiento (por ejemplo a través de un vibrómetro laser) para poder obtener la variación del coeficiente piezoelectrico $\mathrm{d}(\mathrm{E})$ en función del campo eléctrico aplicado (6). Estas medidas muestran que hay una gran correlación entre las dos

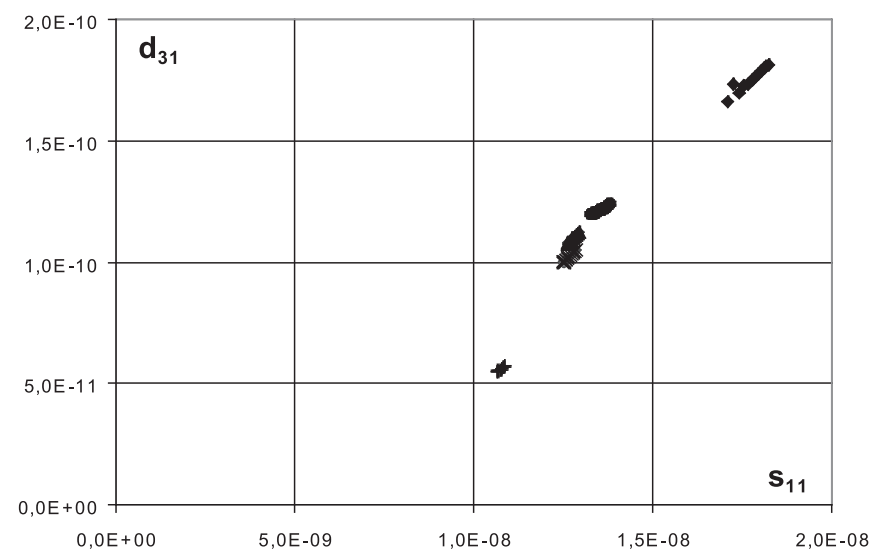

Fig. 1- Comportamiento no lineal de diversas cerámicas comerciales. Representación del coeficiente piezoeléctrico en función del coeficiente elástico, $\mathrm{d}_{31}\left(\mathrm{~s}_{11}\right)$. $\bullet \mathrm{Pz} 27, \bullet \mathrm{Pz} 26, \boldsymbol{\Delta} \mathrm{Pz} 28, \times$ PZT4, + Pz 24. variaciones no lineales, la piezoeléctrica y la dieléctrica, para todos los tipos de materiales.

El otro tipo de no linealidad, ampliamente estudiado por sus repercusiones en los dispositivos resonantes de potencia, es la no linealidad elástica. En este tipo de medidas la variable independiente óptima es el esfuerzo medio $\mathrm{T}$ que actúa en el resonador, obteniéndose las leyes que rigen al coeficiente elástico $\mathrm{s}_{11}(\mathrm{~T})$ o las pérdidas mecánicas $\tan \delta_{\mathrm{m}}(\mathrm{T})$, en función de ese esfuerzo medio T. A pesar de que estas medidas, al realizarse en la resonancia, se llevan a cabo a frecuencias muy superiores (típicamente del orden de $100 \mathrm{kHz}$ ), los resultados obtenidos son muy similares a los obtenidos en el estudio de la no linealidad dieléctrica. Se puede observar que los materiales "blandos" también se rigen por una ley que es similar a la de Rayleigh, siendo la variación del coeficiente elástico proporcional al esfuerzo medio:

$$
\Delta \mathrm{s}_{11}=\mathrm{a}<\mathrm{T}>
$$

siendo a la constante de proporcionalidad, diferente para cada material.

También para estos materiales la relación entre pérdidas mecánicas y variación del coeficiente elástico sigue una proporcionalidad similar a la citada anteriormente, Ec. [3]. En la misma dirección puede decirse que para los materiales "duros" la dependencia con el esfuerzo medio, ya no es lineal sino aproximadamente cuadrática, y que las pérdidas también en este caso son mucho menores que las expresadas en Ec. [3] (7).

\section{1. Tipos de paredes de dominio.}

Vamos a analizar en detalle el movimiento de las paredes de dominio en el efecto extrínseco. Puesto que los dominios ferroeléctricos sólo tienen las posibles polarizaciones que la simetría cristalina del grano permita, también las paredes de dominio, que separan dos dominios contiguos, sólo pueden ser de ciertas categorías, que cumplan las condiciones de contorno eléctricas y mecánicas. Para una perovskita como el PZT, típicamente hay dos tipos de paredes de dominio, las de $180^{\circ}$ y las no- $180^{\circ}$. Estas últimas son de $90^{\circ}$ en el caso de estructura tetragonal o bien de $71^{\circ}$ y $109^{\circ}$ en el caso de los grupos cristalinos romboédricos (8).

Las repercusiones de cada tipo de pared de dominio en las no linealidades son diferentes. Cuando hay un movimiento de las paredes de dominio de $180^{\circ}$, debido a un campo eléctrico exterior (menor al campo eléctrico coercitivo) y a través del efecto extrínseco, cambia la polarización del conjunto del grano, y por lo tanto también el desplazamiento eléctrico y la constante dieléctrica, pero no cambia el tamaño de las celdas ni del grano. Por lo tanto el movimiento de las paredes de dominio en $180^{\circ}$ producirá contribución extrínseca dieléctrica, cambiando la constante dieléctrica $\varepsilon$, pero no contribuirá a la no linealidad del efecto piezoeléctrico d.

En el caso de paredes de dominio de no- $180^{\circ}$ esta diferencia no ocurre, pues al moverse una pared se producen cambios en la polarización y en el tamaño del grano, de forma que se producen contribuciones dieléctricas y piezoeléctricas. Por lo tanto a efectos de aumentar la piezoelectricidad interesará que el número de estos dominios sea máximo, aún a costa de tener mayor no linealidad piezoeléctrica.

Esta diferenciación de comportamientos se ha utilizado para estimar cuantitativamente el tanto por ciento de dominios 
de no- $180^{\circ}$ respecto al número total de dominios $(9,10)$. Esta proporción de cada tipo de dominios varía en función de la composición Zr-Ti de las cerámicas PZT y por lo tanto de la estructura cristalina de la composición, así como de los dopantes aceptores o donores que se añadan.

\section{2. Superposición de efectos y variables independientes.}

Para poder analizar más adecuadamente los efectos intrínseco y extrínseco se acepta, en primer lugar, que estos efectos son independientes y que se pueden superponer. En este estudio se deben identificar cuales son las variables comunes a ambos efectos y que otras variables pueden ser aditivas, como consecuencia de la superposición de los efectos.

Las magnitudes electromécánicas que son comunes a los dos efectos descritos son el esfuerzo $\mathrm{T}$ y el campo eléctrico E. En cambio las magnitudes aditivas, que suponen la superposición de estos efectos, son el desplazamiento eléctrico $\mathrm{D}$ (que al ser proporcional a la carga libre, será la suma de la producida por cada efecto) y la deformación S (también será la suma de la deformación debida a cada efecto). Por lo tanto la ecuación característica que nos interesa utilizar es aquella que depende de las variables independientes T, E. Siendo posible descomponer la matriz de transformación en dos matrices una para el efecto intrínseco y otra para el extrínseco (2). En el caso del efecto extrínseco, puesto que la dependencia deja de ser lineal (dependencia tipo Rayleigh o cuadrática), los valores de $\mathrm{T}$ y E están modificados por funciones $\mathrm{g}(\mathrm{T})$ y $\mathrm{f}(\mathrm{E})$ :

$\left(\begin{array}{l}S \\ D\end{array}\right)=\left(\begin{array}{ll}S & d \\ d & \varepsilon\end{array}\right)\left(\begin{array}{l}T \\ E\end{array}\right)=\left(\begin{array}{ll}s & d \\ d & \varepsilon\end{array}\right)_{\text {int }}\left(\begin{array}{l}T \\ E\end{array}\right)+\left(\begin{array}{ll}s & d \\ d & \varepsilon\end{array}\right)_{\text {ext }}\left(\begin{array}{l}T \cdot g(T) \\ E \cdot f(E)\end{array}\right)$

El uso de estas variables S, D y de los coeficientes s, d y $\varepsilon$ (rigidez, coeficiente piezoeléctrico y constante dieléctrica) es relevante pues, como se ha dicho previamente, permite discriminar ambas contribuciones. La mejor forma de ver el alcance de esta afirmación es el estudio de las gráficas $\mathrm{d}(\varepsilon)$ o bien d(s) (ver Fig. 1 y puntos C-D de la Fig. 2). Si la gráfica se realiza para diferentes valores de la excitación, es decir

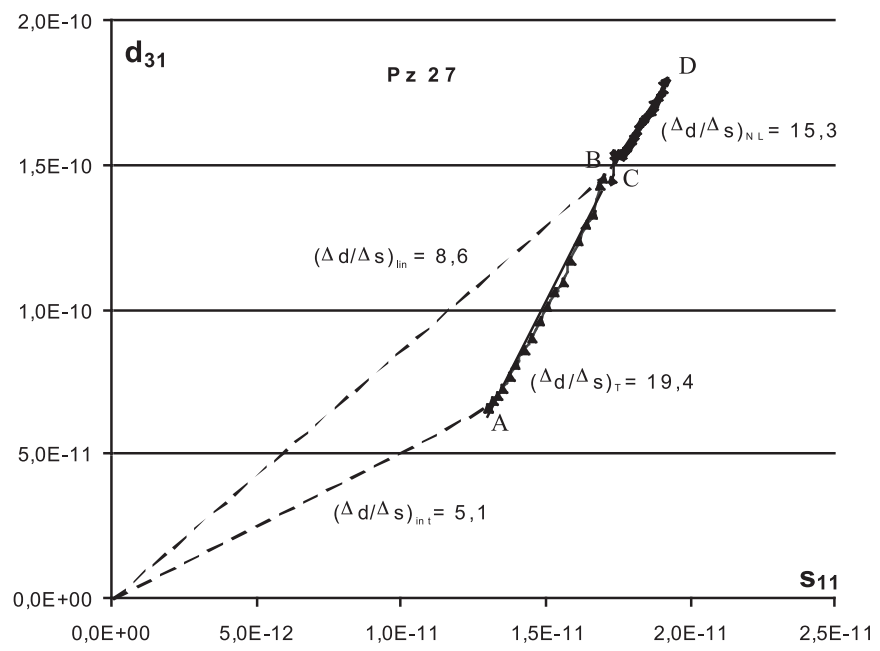

Fig. 2- Comportamiento no lineal (puntos C-D) y variación con la temperatura (puntos A-B), de $\mathrm{d}_{31}\left(\mathrm{~s}_{11}\right)$. Piezocerámica Pz 27. diferentes puntos de las medidas de resonancia no lineales, se puede observar como los valores medidos, corresponden a puntos que se alinean en una recta $(\Delta \mathrm{d} / \Delta \mathrm{s})_{\mathrm{NL}}$ que refleja la contribución extrínseca. En esta gráfica, el calculo de $(\Delta \mathrm{d} / \Delta \mathrm{s})$ ${ }_{\mathrm{NL}}$ se lleva a cabo a partir de las medidas de $\Delta \mathrm{S}$ y $\Delta \mathrm{D}$ en resonancia (es decir para campo eléctrico casi nulo $\mathrm{E}=0$ ), por lo que se obtiene:

$$
\frac{\Delta D}{\Delta S}=\left(\frac{\Delta d}{\Delta s}\right)_{\text {ext }}=\frac{d_{\text {ext }} \cdot T \cdot g(T)}{S_{\text {ext }} \cdot T \cdot g(T)}=\frac{d_{\text {ext }}}{s_{\text {ext }}}
$$

Esta será la primera forma de obtener experimentalmente el valor de los coeficientes extrínsecos asociados a una piezocerámica: las medidas no lineales y su representación adecuada en este tipo de gráfica. Por el contrario, a baja señal (punto $\mathrm{C}$ de la Fig. 2) existen ambos fenómenos: el intrínseco y el extrínseco.

\section{MEDIDAS A MUY BAJA TEMPERATURA}

Un completo análisis del comportamiento de una cerámica ferroeléctrica es el que se obtiene al estudiar el comportamiento dieléctrico, piezoeléctrico y elástico a muy bajas temperaturas (3). Para ello se llevan a cabo medidas lineales tanto de la
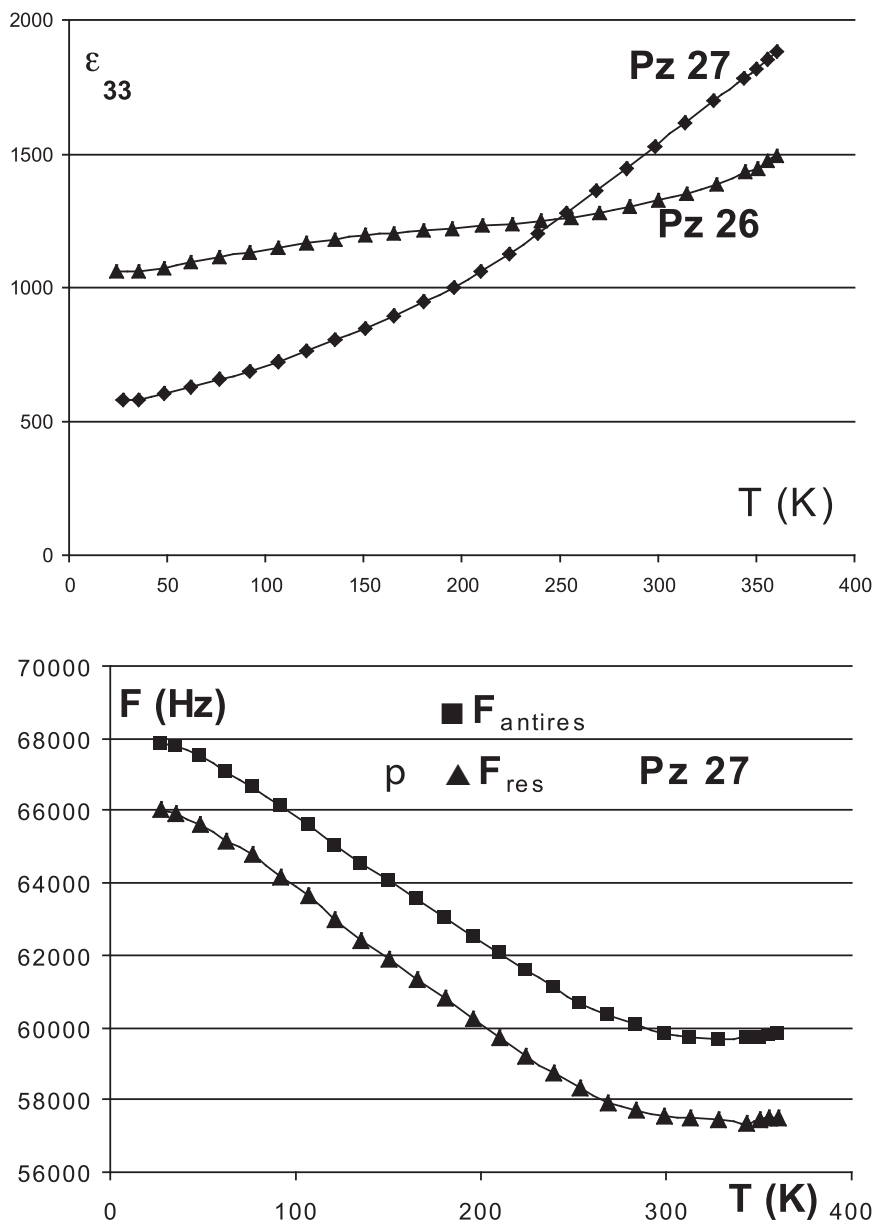

Fig. 3- a) Variación con la temperatura de la permitividad eléctrica relativa $\varepsilon_{33}(\mathrm{Pz} 27, \mathrm{Pz} 26)$ b) Dependencia de las frecuencias de resonancia y antiresonancia con la temperatura ( $\mathrm{Pz} 27)$. 
permitividad dieléctrica como de las frecuencias de resonancia y antiresonancia, en función de la temperatura (Fig. 3). De todas estas curvas se puede decir, en una primera observación, que los coeficientes dieléctricos y piezoeléctricos disminuyen al bajar la temperatura.

En este tipo de análisis se establecen un conjunto de hipótesis que es necesario predefinir y verificar a posteriori. Se acepta que el efecto intrínseco es un efecto lineal, por lo que no está presente en las observaciones no lineales con alto campo aplicado. También se acepta que el efecto intrínseco no varía sensiblemente con la temperatura, siempre que nos mantengamos aceptablemente lejos de las transiciones de fase ferroeléctrica. Asimismo la polarización de la cerámica casi no varía al bajar la temperatura (lejos de las transiciones de fase), por lo que apenas afectará al efecto intrínseco (3).

La hipótesis sobre el efecto extrínseco es la siguiente. Es de esperar que a muy baja temperatura, en la proximidad de los $0 \mathrm{~K}$, los dominios queden congelados, perdiéndose toda la movilidad de las paredes de dominio. Por lo tanto se hace la hipótesis de que a esas bajas temperaturas, el efecto extrínseco se anula.

Consecuencia de todo lo anterior, es que el efecto extrínseco es la causa de las no linealidades. En cuanto a la variación con la temperatura, podemos decir que afectará únicamente a la contribución extrínseca.

De la conjunción de las hipótesis sobre ambos efectos surge la posibilidad de interpretar, a través de la gráfica $\mathrm{d}(\mathrm{s})$ citada anteriormente, ambas caracterizaciones: la no linealidad y la variación con la temperatura. Esto es lo que se muestra en la figura 2. Los puntos C-D reflejan las medidas obtenidas con diferentes niveles de excitación, es decir no lineales, y por lo tanto su pendiente refleja la contribución extrínseca. Los puntos B-A representan las medidas lineales para diferentes temperaturas, desde la temperatura ambiente (punto B, que coincide con el $\mathrm{C}$ al ser a la misma temperatura y ambos correspondiendo a baja señal) hasta el punto A, a muy baja temperatura, próxima a $0 \mathrm{~K}$. La pendiente de los puntos de esta serie de medidas B-A corresponde también al efecto extrínseco $\mathrm{e}_{\mathrm{ext}}=(\Delta \mathrm{d} / \Delta \mathrm{s})_{\mathrm{T}}$, en este caso debido al efecto de la temperatura.

La comparación entre estas dos pendientes, la producida en C-D por efecto no lineal, $\mathrm{e}_{\mathrm{ext}}=(\Delta \mathrm{d} / \Delta \mathrm{s})_{\mathrm{NL}}$, $\mathrm{y}$ la producida en B-A, por la variación con la temperatura $e_{\text {ext }}=(\Delta \mathrm{d} / \Delta \mathrm{s})_{\mathrm{T}}$, muestra que existe una buena concordancia entre ambas formas de medir el efecto extrínseco. Con ello queda aceptablemente verificado el conjunto de hipótesis anteriores.

En la figura 2, puede además observarse que a muy baja temperatura, si el efecto extrínseco ha desaparecido, solo queda el efecto intrínseco, que será posible medir por esta vía. Concretamente puede obtenerse el cociente intrínseco $\mathrm{e}_{\text {int }}=(\mathrm{d} / \mathrm{s})_{\text {int }}$, que es el menor de los descritos. Se observa también, que a temperatura ambiente y baja señal, puntos B y C coincidentes, el valor de los coeficientes lineales d y s son la suma de una contribución intrínseca y otra extrínseca, pudiendo calcular el cociente lineal $\mathrm{e}_{0}=(\mathrm{d} / \mathrm{s})_{0}$.

\section{ANISOTROPIA DEL EFECTO EXTRINSECO}

Para completar todos los estudios anteriores se ha desarrollado un modelo que tiene en cuenta la estructura de los dominios ferroeléctricos en estas cerámicas (8). La observación de estos dominios (11-13), muestra en muchos casos una morfología laminar en el interior de cada grano cerámico, de forma que cada lámina es un monodominio separado de su vecino por paredes de dominio de no- $180^{\circ}$. Estas paredes de dominio se presentan como planos paralelos separados por una distancia d que es casi la misma para todos ellos (dependiendo sólo del tamaño del grano (14)) (Fig. 4).

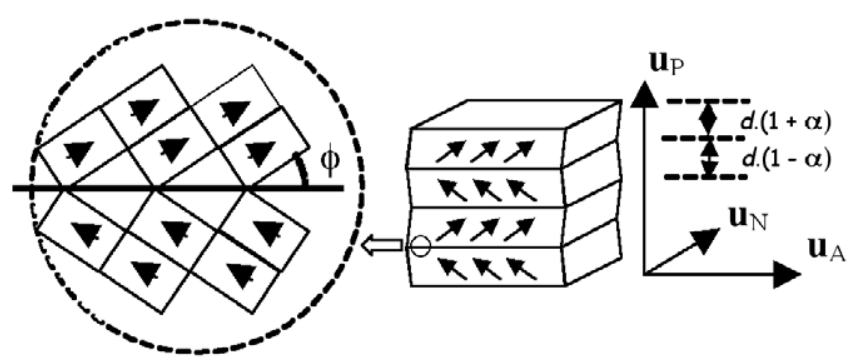

Fig. 4- Estructura laminar de dominios ferroeléctricos, con paredes de dominio de no- $180^{\circ}$, y ejes de coordenadas adoptados $\left\{u_{\mathrm{p}^{\prime}} \mathrm{u}_{\mathrm{A}^{\prime}}, \mathrm{u}_{\mathrm{N}}\right\}$.

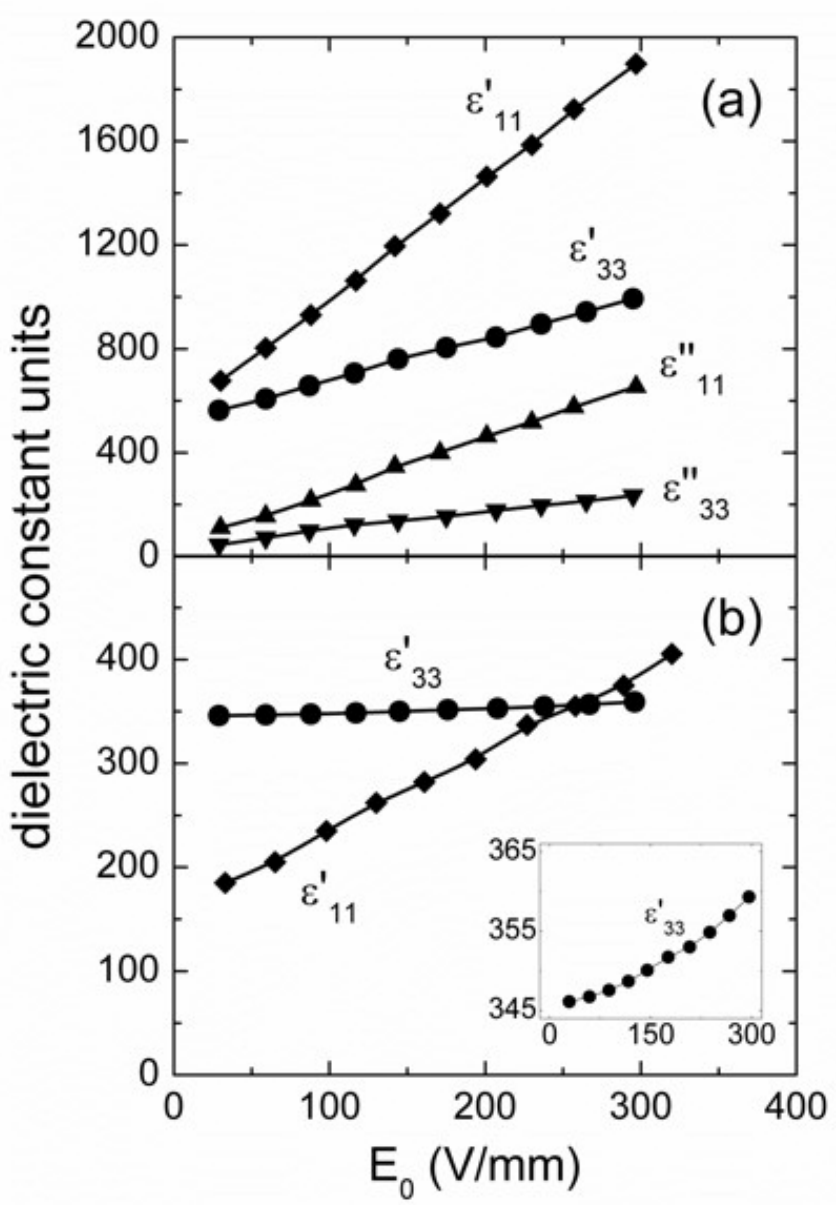

Fig. 5- Anisotropía de la permitividad eléctrica relativa. Variación de $\varepsilon_{33}^{\prime}$ y $\varepsilon^{\prime}{ }_{11}$ en función del campo eléctrico aplicado, así como de sus partes imaginarias (pérdidas) $\varepsilon^{\prime \prime}{ }_{33}, \varepsilon^{\prime \prime}{ }_{11}$. a) Soft PZT. b) Hard PZT.

Se pueden asignar unos ejes vinculados a esta configuración, de forma que el eje perpendicular a los planos de las paredes de dominio, $\mathrm{u}_{\mathrm{p}}$, será el de la dirección de la polarización de este conjunto. El eje contenido en el plano de la paredes y que contiene la proyección positiva o negativa de la 
polarización de los dominios, $\mathrm{u}_{\mathrm{A}^{\prime}}$ corresponde a la dirección activa, ya que cuando se aplique el campo eléctrico en esta dirección es cuando moveremos las paredes, haciendo crecer a los dominios que están en la dirección del campo, a costa de los otros dominios que disminuirán. Por último la dirección perpendicular a las anteriores, contenida en las paredes y perpendicular a la polarización de los dominios, $\mathrm{u}_{\mathrm{N}^{\prime}}$ es la dirección neutra: en ella no se alteran para nada las polarizaciones ni las paredes al aplicarse un campo o esfuerzo en esa dirección.

Analizando lo que ocurre en un sistema como el descrito, puede deducirse que si se aplica un campo eléctrico en la dirección de la polarización, $\mathrm{u}_{\mathrm{p}^{\prime}}$ no se favorece ningún conjunto de los dominios respecto al otro. En cambio un campo eléctrico aplicado en la dirección perpendicular a la de la polarización, en la dirección activa, $\mathrm{u}_{\mathrm{A}^{\prime}}$ si que producirá un importante movimiento de las paredes de dominio y por lo tanto efecto extrínseco.

Para una cerámica hay que tener en cuenta que los granos tienen estas estructuras con los vectores $u_{p^{\prime}}$ en direcciones próximas, pero no coincidentes, a las de la polarización de la cerámica. Para poder contrastar este resultado se ha medido la no linealidad de la permitividad dieléctrica $\varepsilon_{33}$ de cerámicas en la dirección de la polarización, así como la no linealidad de la permitividad $\varepsilon_{11}$, en la dirección perpendicular a la polarización. La figura 5 muestra estos resultados. Puede observarse como la permitividad dieléctrica $\varepsilon_{11}$ es mayor que la $\varepsilon_{33}$ y que la no linealidad, debida al efecto extrínseco, es también mucho mayor. Además existe una dependencia con el campo eléctrico para la permitividad $\varepsilon_{11}($ E) que cumple la ley de Rayleigh, a pesar de que este material no la cumplía para $\varepsilon_{33}(\mathrm{E})$, es decir la no linealidad en la dirección activa si que verifica la ley de Rayleigh. También se observa que la no linealidad en la permitividad $\Delta \varepsilon_{11}$ es del mismo orden de magnitud tanto para las cerámicas blandas como para las duras.

Otra observación posible es que la permitividad dieléctrica es mayor en la cerámica no polarizada que en la cerámica polarizada. Este resultado es acorde con el modelo expuesto, ya que al polarizarla es cuando se crea la configuración anterior en cuyo caso el efecto extrínseco es menor en la dirección de polarización que en la dirección perpendicular a la polarización (sería nulo para aquellos granos donde coincida la dirección $\mathrm{u}_{\mathrm{p}}$ con la dirección del campo aplicado). Esto no ocurre en la cerámica no polarizada, pues al poder existir en ese modelo direcciones de polarización del grano al azar, un campo eléctrico fácilmente puede coincidir con la dirección activa de varios granos, sobre los que producirá efecto extrínseco.

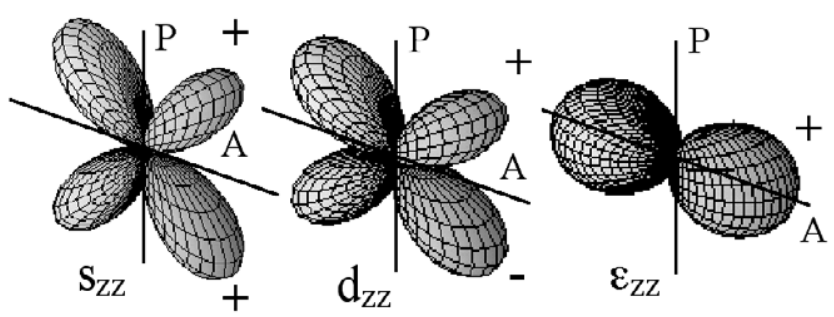

Fig. 6-Contribución extrínseca de los coeficientes elástico, piezoeléctrico y dieléctrico, de un monocristal a lo largo de la dirección $Z$, asociada a la dirección del campo eléctrico o esfuerzo aplicados, en función de dicha dirección.
Es de destacar que esta asimetría se refiere al efecto extrínseco únicamente, mientras que el efecto intrínseco tendrá la asimetría propia de la estructura cristalina de la cerámica. Paralelamente se ha utilizado el modelo anterior para analizar el efecto del comportamiento extrínseco sobre el coeficiente piezoeléctrico y el elástico. La figura 6 muestra la variación del efecto extrínseco en cada caso, para el caso de monocristales

\section{CONCLUSIONES}

La comprensión de la compleja microestructura de las piezocerámicas, formada por dominios ferroeléctricos, se aborda a través de dos vías. Por un lado gracias al estudio de las no linealidades dieléctricas, piezoeléctricas y elásticas, por otro lado a través del estudio de las características lineales a muy baja temperatura. Se ha comprobado que hay una aceptable concordancia entre los resultados obtenidos por ambos métodos.

Se ha desarrollado un modelo, que contempla la estructura laminar de la mayoría de las cerámicas piezoeléctricas, y que permite comprender la anisotropía que aparece en el efecto extrínseco. Este modelo facilita la comprensión de muchas de los fenómenos anisótropos que poseen estas cerámicas.

El efecto extrínseco, debido al movimiento de las paredes de dominio, es el que produce un incremento considerable de las propiedades dieléctricas y piezoeléctricas de las piezocerámicas. Se ha analizado que magnitudes pueden ser sumadas al superponer este efecto extrínseco al intrínseco.

Es de destacar que una gran contribución del efecto extrínseco supone un elevado valor para los coeficientes dieléctricos y piezoeléctricos, pero en contrapartida también genera una mayor no linealidad en su comportamiento con alta señal. Es preciso optar por un compromiso en función de la aplicación deseada. Para aplicaciones de potencia se utilizarán cerámicas con menor efecto extrínseco, mientras que para detectores de señal o sensores generalmente interesará materiales con elevados coeficientes y efecto extrínseco.

Las herramientas que se proponen aparecen como indispensables en el estudio de nuevas cerámicas, pues permiten conocer las aportaciones intrínsecas y extrínsecas. En las actuales circunstancias, de búsqueda de nuevas cerámicas sin plomo, estas herramientas deben facilitar la optimización de las cerámicas para cada tipo de aplicación.

\section{AGRADECIMIENTOS}

Este artículo ha sido posible gracias al proyecto español MAT2004-01341 (CICYT), a los fondos europeos FEDER y a la red europea POLECER (G5RT-CT-2001-05024).

\section{BIBLIOGRAFÍA}

1. Damjanovic D., Denmartin M., Contribution of the irreversible displacement of domain walls to the piezoelectric effect in barium titanate and lead zirconate titanate ceramics, J. Phys.: Condens. Matter., 9, 4943 (1997).

2. Perez R., Albareda A., Garcia J. E., Tiana J., Ringgaard E., Wolny W., Extrinsic contribution to the non-linearity in a PZT disc, J. Phys. D: Appl. Phys., 37. 2648-2654 (2004)

3. Zhang Q.M., Wang H., Kim N., Cross L.E., Direct evaluation of domain-wall and intrinsic contributions to the dielectric and piezoelectric response and their temperature dependence on lead zirconate-titanate ceramics, J. Appl. Phys., 75. 454-459 (1994). 
4. Hall, D., Stevenson P., High field dielectric behaviour of ferroelectric ceramics, Ferroelectrics, 228, 139 (1999).

5. Garcia J.E., Perez R, Albareda A., Contribution of reversible processes to the non-linear dielectric response in hard zirconate titanate ceramics, J. Phys.: Condens. Matter, 17, 7143-7150 (2005).

6. Andersen B., Ringgaard E., Bove T., Albareda A. and Perez R., Performance of piezoelectric ceramic multilayer components based on hard and soft $\mathrm{PZT}$, Actuator 2000: Proc. 7th International Conf. on New Actuators, 419-422 (2000).

7. Albareda A., Pérez R., Casals J.A., García J.E., Ochoa D.A., Optimization of Elastic Nonlinear Behavior Measurements of Ceramic Piezoelectric Resonators with Burst Excitation, IEEE Trans. on Ultras. Ferroel. Freq. Control, 54, 10, 2175-2188 (2007).

8. Pérez R., García J.E., Albareda A., Ochoa D.A., Extrinsic effects in twinned ferroelectric polycrystals, J. Appl. Phys., 102, 044117 (2007)
9. Albareda A., Pérez R., García J.E., Tiana J., Pérez E., Gorri J.A., Extrinsic coefficient characterisation of PZT ceramics near the morphotropic phase boundary, Bol. Soc. Esp. Ceram. V., 45, 3, 184-187 (2006).

10. Albareda A., Pérez R., García J.E., Ochoa D.A., Gomis V., Eiras J.A., Influence of donor and acceptor substitutions on the extrinsic behaviour of PZT piezoceramics, J. of Eur. Ceram. Soc., 27, 4025-4028 (2007).

11. B. Jaffe, W.R. Cook, H. Jaffe. Piezoelectric Ceramics (London:Academic, 1971)

12. Rabe U., Kopycins, Hirsekorn, Muñoz-Saldaña, Schneider, Arnold, High resolution characterisation of piezoelectric ceramics by ultrasonic scanning force microscopy techniques, J. Phys. D: Appl. Phys., 35, 2621 (2002)

13. Erhard J., Cao W., Effective material properties in twinned ferroelectric crystals, J. Appl. Phys., 86, 1073 (1999).

14. Arlt G. and Pertsev N. A., Force constant and effective mass of $90^{\circ}$ domain wall in ferroelectric ceramics, J. Appl. Phys., 70 , 2283-2289 (1991 ). 